\title{
LOW FREQUENCY NOISE IN TANTALUM CAPACITORS
}

\author{
D.T. SMITH \\ University of Oxford, Clarendon Laboratory, Parks Road, Oxford OX1 3PU.
}

Noise has been measured in a number of biased solid tantalum capacitors at frequencies down to $0.01 \mathrm{~Hz}$. The noise current was found to have a $1 / f$ power spectrum, and the amplitude varied with the bias voltage with a law in the range 1st to 4 th power. There was a large difference in amplitudes between different capacitors of the same type.

\section{INTRODUCTION}

Capacitors are used in low-frequency, low-noise systems to couple signals, while blocking d.c. levels, or to decouple, where a short circuit at signal frequencies is needed to prevent unwanted signal paths. It has been found that capacitors sometimes generate spurious signals, or noise, which will degrade the performance of the system.

Ideally, capacitors are loss free components, and therefore also noise free. In practice, there are a number of loss mechanisms, and so noise can be generated when a capacitor is not in equilibrium, and in particular when it is biased. Plastic dielectric capacitors probably have the lowest noise at low frequencies, but they become large and expensive because of the high values needed at low frequencies. While electrolytic capacitors are small and cheap, aluminium electrolytics have been found to be very noisy at low frequencies.

The object of the work described here was to look at the noise in some commercial tantalum electrolytic capacitors ${ }^{1,2}$ to collect some quantitative data, to find how the noise varied with operating conditions, to compare capacitor types, and if possible to find guidelines for keeping this noise to a minimum.

\section{MEASUREMENT PROCEDURE}

Measurements were made in the first instance on capacitors of one value and working voltage $(47 \mu \mathrm{F}, 16 \mathrm{~V})$, as this enabled direct comparisons to be made. Later tests were made on different voltage capacitors, and other capacitance values were used as necessary. Initially, all devices were measured at $9 \mathrm{~V}$, but other bias voltages were used later.

The basic circuit used is shown in figure 1 . For $9 \mathrm{~V}$ measurements, an alkaline battery (MN1604) was used with a $4.7 \mathrm{M} \Omega(2 \mu \mathrm{A})$ bleed resistor, as this gave a lower noise than supplies using Zener diodes. Combinations of alkaline batteries were used to give other voltages as required. As there was a significant leakage in many of the capacitors being tested, a significant voltage was developed across $\mathrm{R} 1$. This would have saturated the amplifier if it had been applied directly to the amplifier input. A second (polyester) capacitor, $\mathrm{C} 2$, was used to block the bias. The diodes limited the input voltages to protect the amplifier, and R3 limited currents to safe values, when the capacitor under test was replaced and the new capacitor was charging. As $\mathrm{C} 2$ is a plastic dielectric type and operated at a low bias, it did not add a significant extra noise. The amplifier had a low noise FET input, but its noise was still significant and had to be subtracted to find the capacitor noise.

To keep external interference to a minimum, the capacitor under test, bias supply and the first two amplifier stages were enclosed in an assembly of screened boxes, and supplied from 


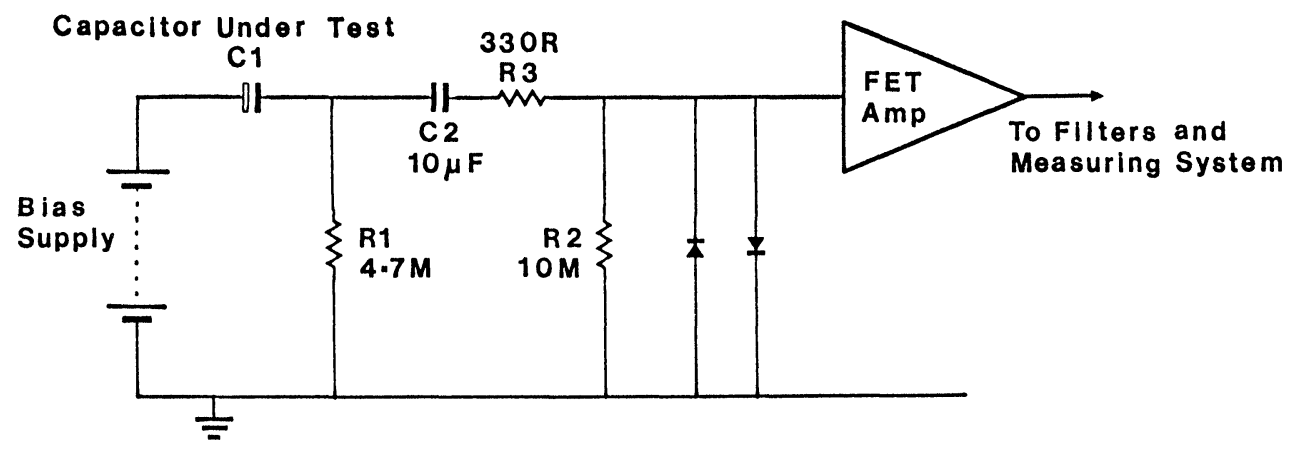

FIGURE 1. Circuit used for measurements.

a remote mains-powered source. The box containing the capacitor was temperaturecontrolled, and held at $25 \mathrm{C}$.

After further amplification, the noise was fed to a group of filters ${ }^{3}$ with a $Q$ of $1 / 2$ at frequencies of $0.01,0.1,1$ and $10 \mathrm{~Hz}$, and then on to a data collection microprocessor. The amplifier and system noise was measured with the capacitor at zero bias (and so noise free), so that it could be subtracted from the total noise to find the noise of the biased capacitor. Long measuring times were necessary because of the low frequencies, and most measurements lasted for about one day.

Capacitors from six manufacturers were measured. All were polarised solid tantalum types, with working voltages of 10 to 35 .

\section{MEASUREMENTS}

\subsection{Reproducibility of measurements}

Measurements were made on given samples on different occasions, at the same bias and temperature. Some later measurements were made after the sample had been held at the working bias for periods of over two weeks.

The measured noise levels varied a little, and tended to be lower on the second occasion. This change was normally less than a ratio of $2: 1$, which was less than the typical sample-tosample change for components of the same type.

\subsection{Variations between samples of the same type}

Figure 2 shows the measured noise levels at $0.01 \mathrm{~Hz}$ with $9 \mathrm{~V}$ bias for 32 samples of $47 \mu \mathrm{F}$, $16 \mathrm{~V}$ bead capacitors from five manufacturers. This show a range of variation of measured values for a given manufacturer from 7:1 (E) to over 300:1 (B). It also shows significant differences between manufacturers, with $\mathrm{D}$ having the lowest noise at $9 \mathrm{~V}$ bias. Two samples had a noise level too small to measure with the amplifier used: these are marked ' $x$ ' in figure 2 .

\subsection{Measured noise frequency spectrum}

All the devices measured had a current noise power spectrum which was approximately $1 / \mathrm{f}$ within the range of measurement 0.01 to $10 \mathrm{~Hz}$. This was a repeatable feature of the measurements, and probably holds over a wider frequency range. Figure 3 shows some measured spectra which were typical of the results obtained. 


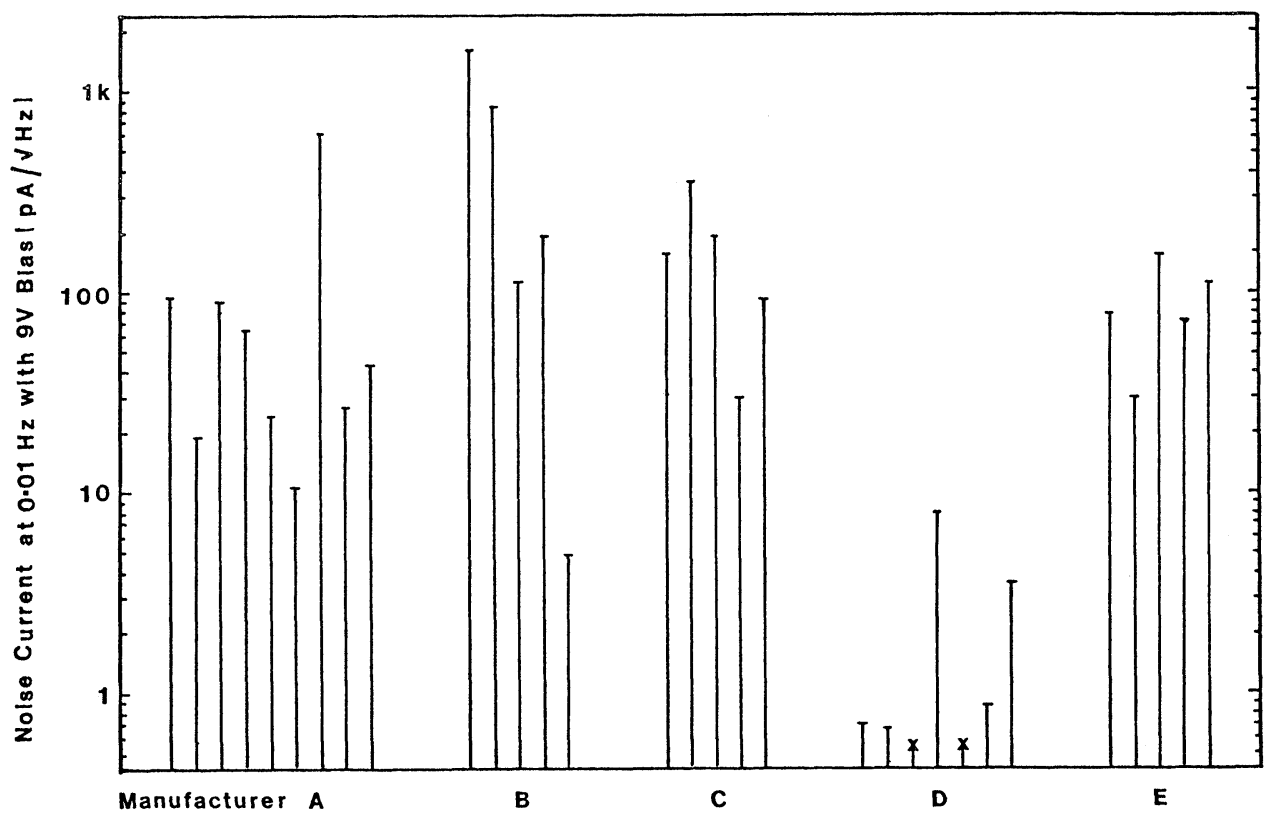

FIGURE 2. Measured noise currents of 32 sample bead capacitors, all $47 \mu \mathrm{F}, 16 \mathrm{~V}$ working at $9 \mathrm{~V}$ bias.

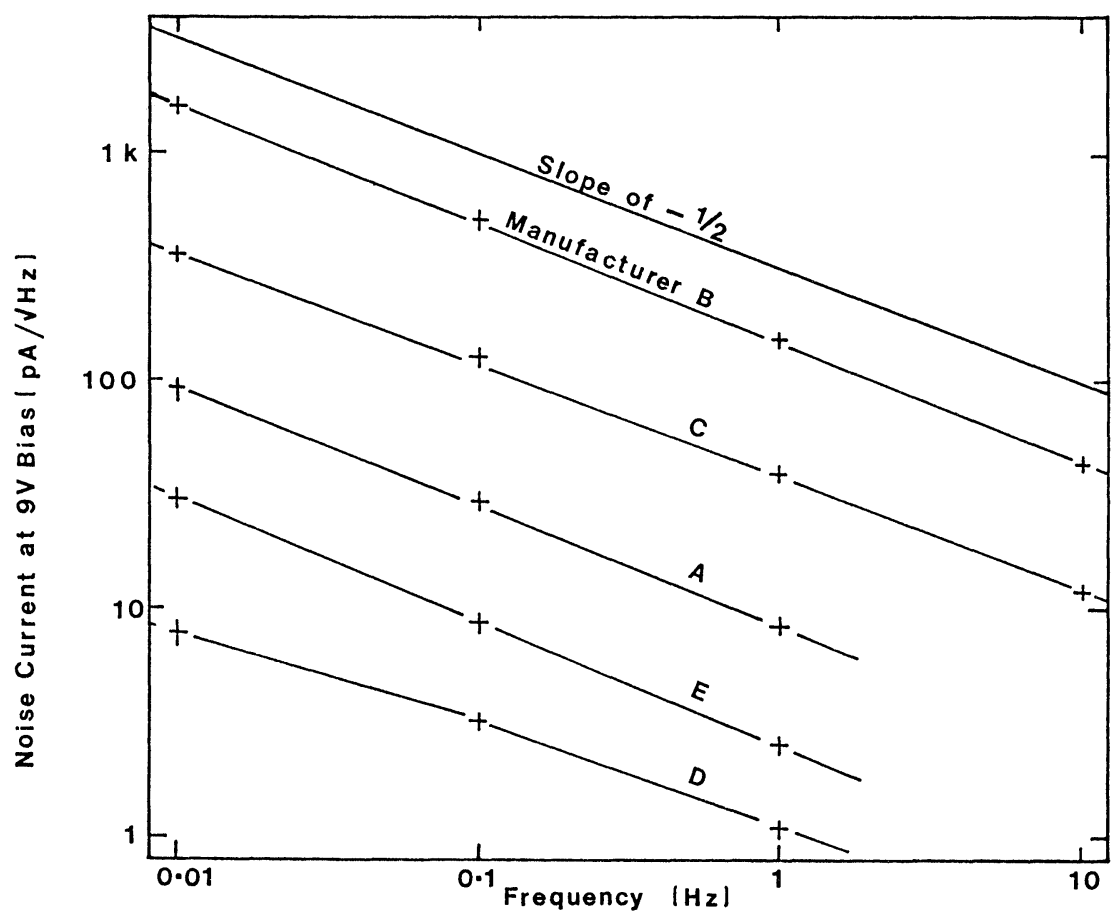

FIGURE 3. Variation of noise current of 5 sample capacitors with frequency. 
Assuming a power law of the form $S(f)=\mathrm{f}^{-\alpha}$, the value of $\alpha$ was calculated for all runs giving measurable noise in the range from 0.01 to $1 \mathrm{~Hz}$. These values had a mean value of $\alpha$ of 1.025, with a standard deviation of 0.098 , and minimum and maximum values of 0.71 and 1.24. The voltage noise fell sharply with increasing frequency with a power law of approximately $1 / \mathrm{f}^{3}$. With the equipment used, $10 \mathrm{~Hz}$ was a practical upper limit for measurements.

In this article, noise currents are generally quoted for $0.01 \mathrm{~Hz}$ which was the lowest frequency of measurement, as the noise was greatest and most easily measured at this frequency.

\subsection{Variation of noise with capacitor bias}

If the noise generating mechanism was linear, a linear relation would be expected between bias voltage and rms noise current. Figures $4 \mathrm{a}$ and $4 \mathrm{~b}$ show the variation of noise current with bias voltage at $0.01 \mathrm{~Hz}$, in two capacitors showing very different characteristics. Figure 4a shows results for a capacitor (manufacturer A) with an approximately linear relation between noise and bias voltage. This was typical of a number of capacitors from this manufacturer in particular, and a few others from elsewhere. Figure $4 \mathrm{~b}$ shows results for a capacitor from manufacturer D where the noise current increases almost with the 4th power of bias. This, too, was typical of a number of samples. Results generally showed that the rms noise current varied between 1 st and 4th power of the bias voltage (with extremes of 0.8 and 4.3 power), with the range 2 nd to 4 th power being most common. Results on a log-log plot generally gave an approximately straight line, indicating a constant power law relation, but there were some exceptions.

The power law of A's capacitors was usually lower than that of other makes. This suggests that A's capacitors may have lower noise levels at higher bias voltages.
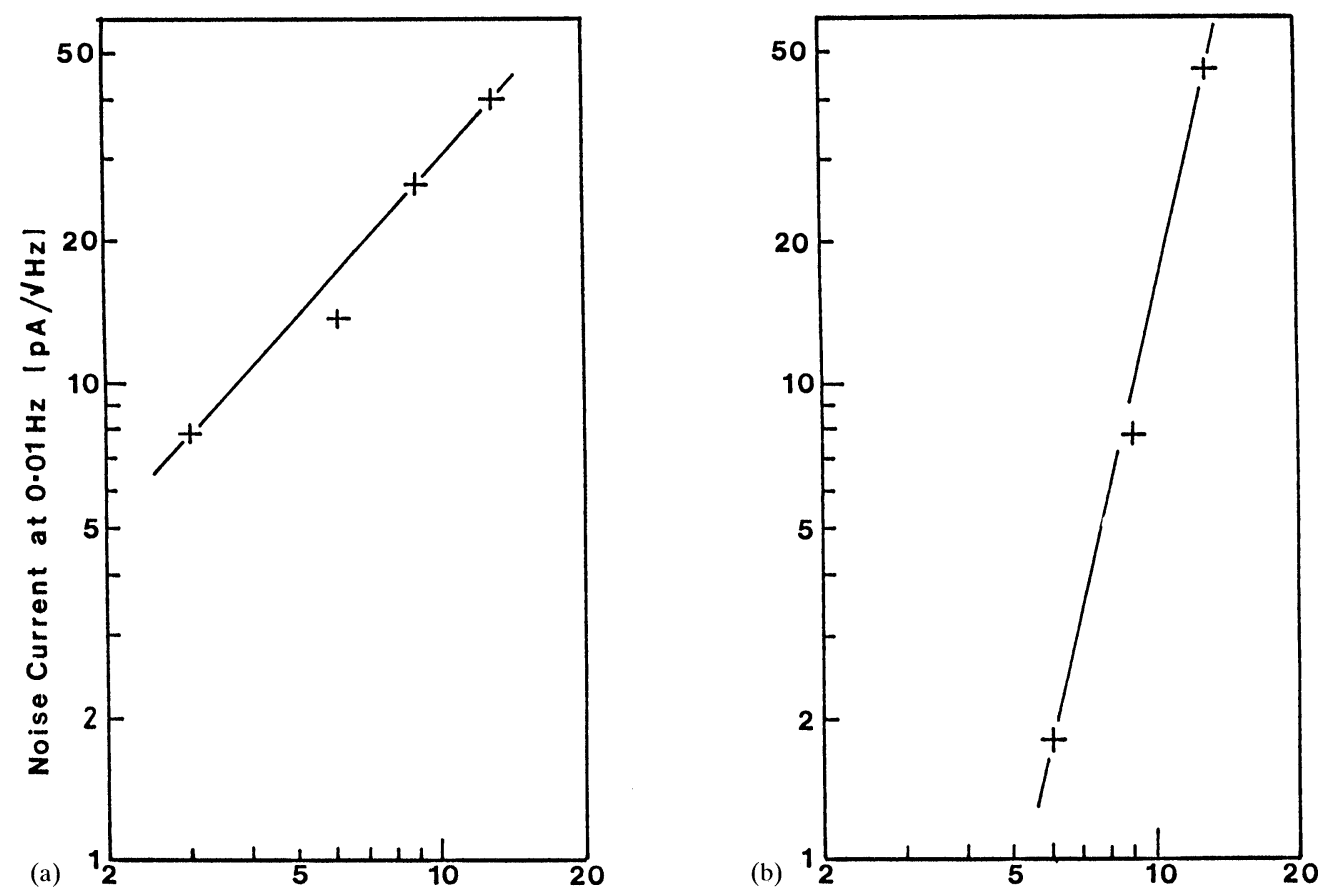

FIGURE 4. Variation of noise current of 2 sample capacitors with bias voltage. 


\subsection{Variation with capacitor voltage rating}

As was expected, the noise observed at a given bias was generally lower for capacitors with a higher voltage rating. Figure 5 shows the (geometric) mean noise currents of groups of capacitors (manufacturer A) plotted against the rating. This shows a general fall in noise with rated voltage. However, the $10 \mathrm{~V}$ rated capacitors showed a lower noise than the $16 \mathrm{~V}$ capacitors. This anomaly is presumably due to the small statistical sample sizes.

\subsection{Correlation between noise current and D.C. leakage}

Direct measurement of capacitor noise is tedious and needs specialised equipment, but d.c. leakage can readily be measured with simple equipment. It was thought that there might be a correlation between the magnitudes of the noise and leakage currents, and if this was strong enough an estimate of noise could be made from a measurement of the leakage.

This relation was therefore investigated, and a correlation was found. It was found to be of some use for evaluating noise performance.

The values of leakage current and noise current for all the 81 runs for which suitable data is available are collected in figure 6 . This shows points scattered about a line of slope approximately +1 .

The currents were converted into logarithms because of the wide range of values, and then analysed. The 81 runs shown in figure 6 gave a (geometric) mean noise current of $15.3 \mathrm{pA} / \mathrm{Hz}^{1 / 2}$ at $0.01 \mathrm{~Hz}$, mean leakage current of $9.14 \mathrm{nA}$, a correlation coefficient of 0.75 , and a mean slope of 1.05 .

Figure 6 shows that of the 81 points, 8 have noise currents that differ by a factor of 10 or more from the line of mean slope. The largest deviation from the best straight line fit was a factor of 37. The standard deviation of the logarithm (to base e) of noise from the line was 1.47 - i.e. a factor of $\operatorname{Exp}(1.47)$ or 4.34.

If capacitor noise cannot be measured directly, measurement of the d.c. leakage can be a useful indicator of the probable noise level.

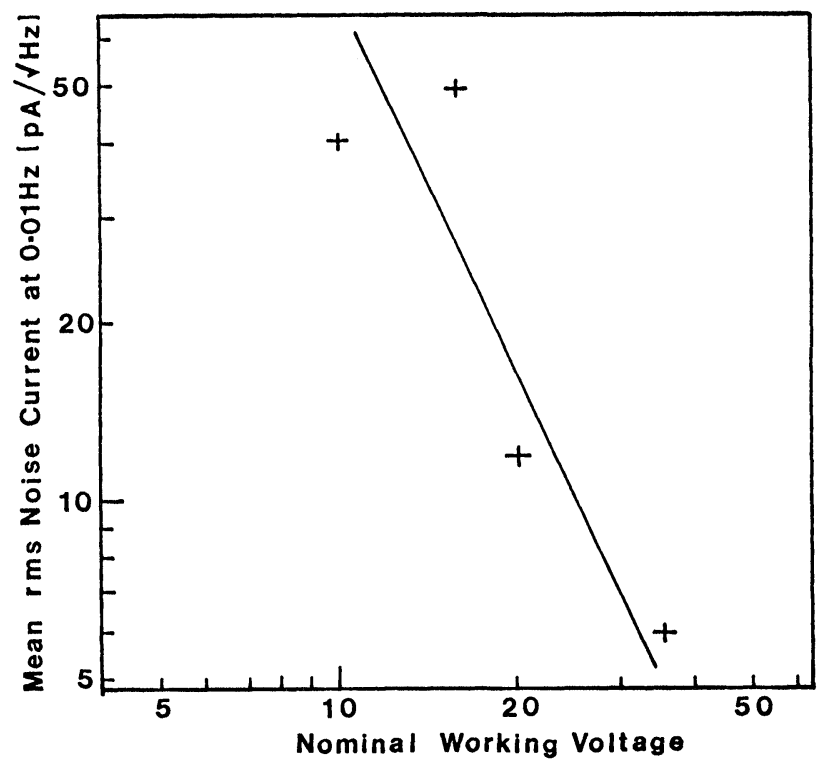

FIGURE 5. Variation of mean noise current with rated working voltage for capacitors from manufacturer A. 


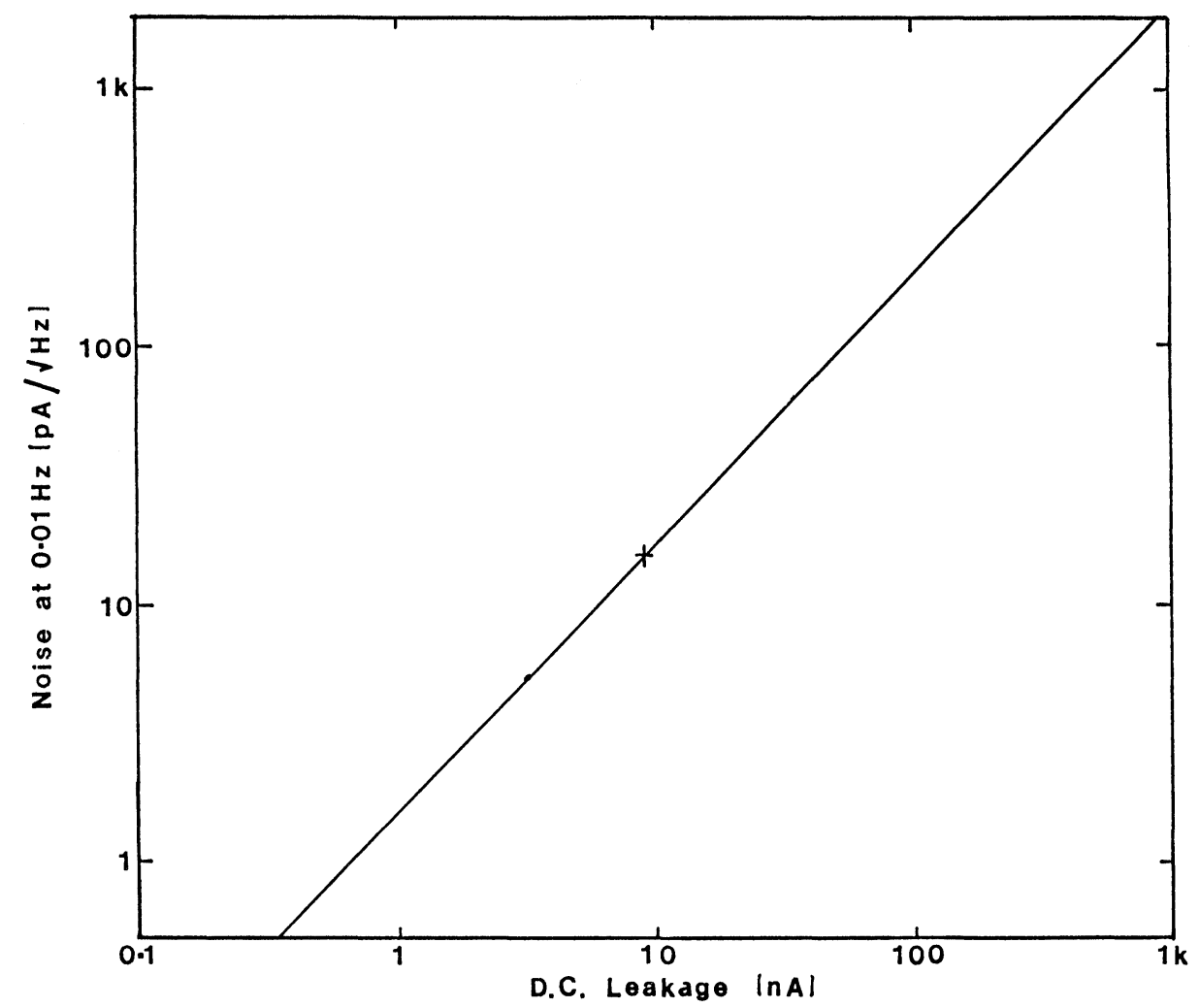

FIGURE 6. Plot of leakage current against rms noise current at $0.01 \mathrm{~Hz}$ of all capacitors measured.

\section{CONCLUSIONS AND RECOMMENDATIONS}

Not too much should be read into these results because the number of samples was limited. However, the following general points emerge:

i) When tantalum capacitors are biased they generate significant levels of noise, and this should be considered when they are used in low-frequency, low-noise applications.

ii) The current noise power spectrum of a biased tantalum capacitor is approximately $1 / \mathrm{f}$ between 0.01 and $10 \mathrm{~Hz}$, and probably over a wider range of frequencies. The voltage noise is therefore $1 / \mathrm{f}^{3}$ when the capacitor is not loaded by an external circuit.

iii) The noise of a particular sample can be expected to remain stable within a factor of two or so when used under constant conditions. However, there are bigger differences between different samples of the same type, and between different makes of outwardly similar capacitors.

iv) The noise varies strongly with the applied bias. The relation between the observed noise current and the applied bias varied between linear and 4th power for the samples measured.

v) There is some correlation between the magnitudes of the leakage current and the noise current. A small d.c. leakage indicates that the noise is probably low. 
vi) For minimum noise the bias should be kept as low as possible, and capacitors with high working voltages used.

vii) For minimum noise, the choice of capacitor manufacturer is important. At $9 \mathrm{~V}$ bias, manufacturer D's capacitors were the quietest measured, but at higher voltages A's may be quieter because of the different rates of increase with bias.

\section{ACKNOWLEDGEMENTS}

The work was supported by the Procurement Executive, Ministry of Defence (Directorate of Components, Valves and Devices) U.K., and supported by the Royal Signals and Rescarch Establishment (R.S.R.E.).

\section{APPENDIX}

The capacitors measured were manufactured by Dubilier, N.E.C., Sprague, S.T.C., Tompson C.S.F. and Waycom.

\section{REFERENCES}

1. J.A. Sunda, "The Application of Tantalum Electrolytic Capacitors." Waycom Technical Publications (1977).

2. R.F. Leech, "The Production of Solid Tantalum Capacitors." The Production Engineer, 44, 349, (1965).

3. D.T. Smith, "Filters for very low frequency noise measurements." Int. J. Electronics, 60, 577 (1986). 

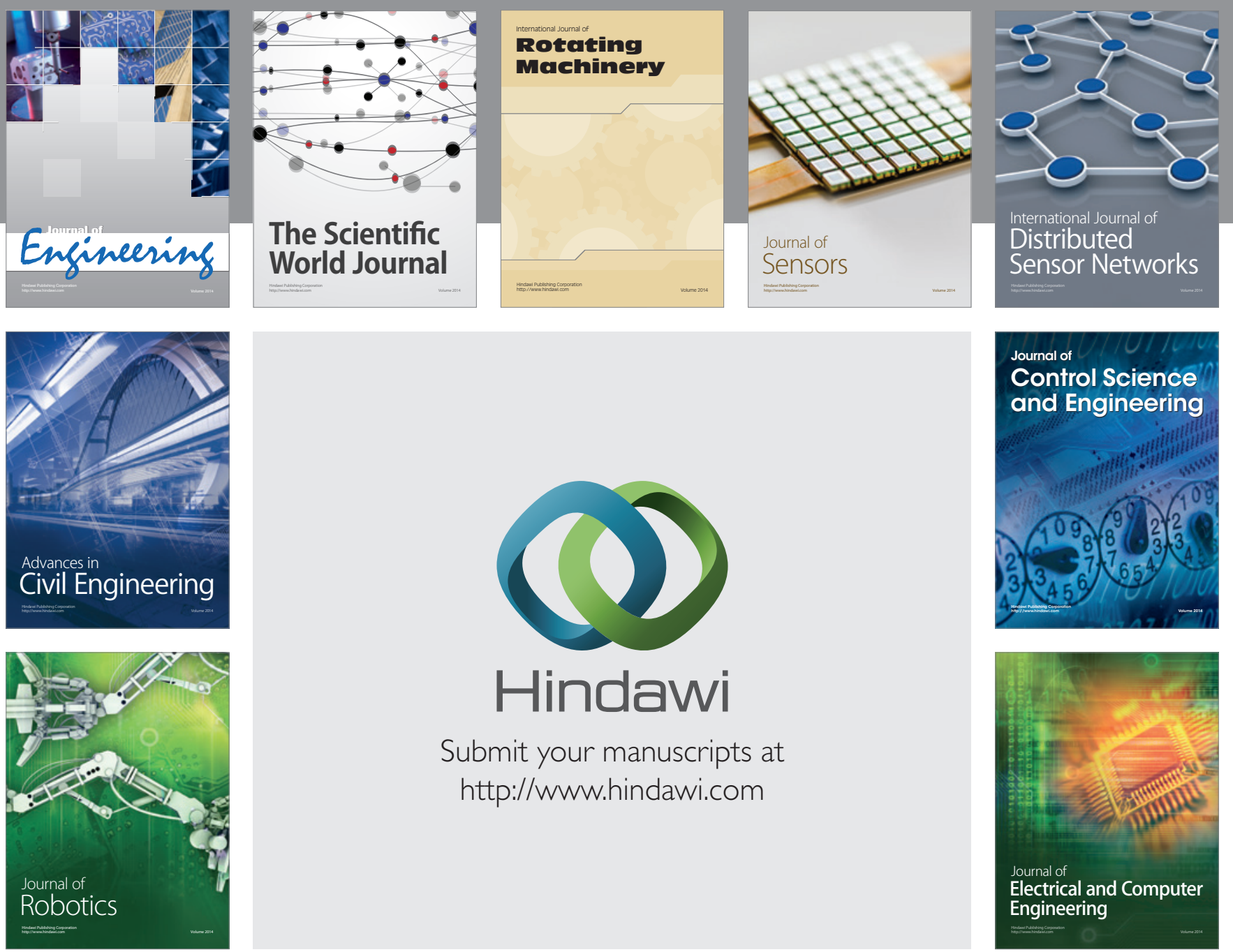

Submit your manuscripts at

http://www.hindawi.com
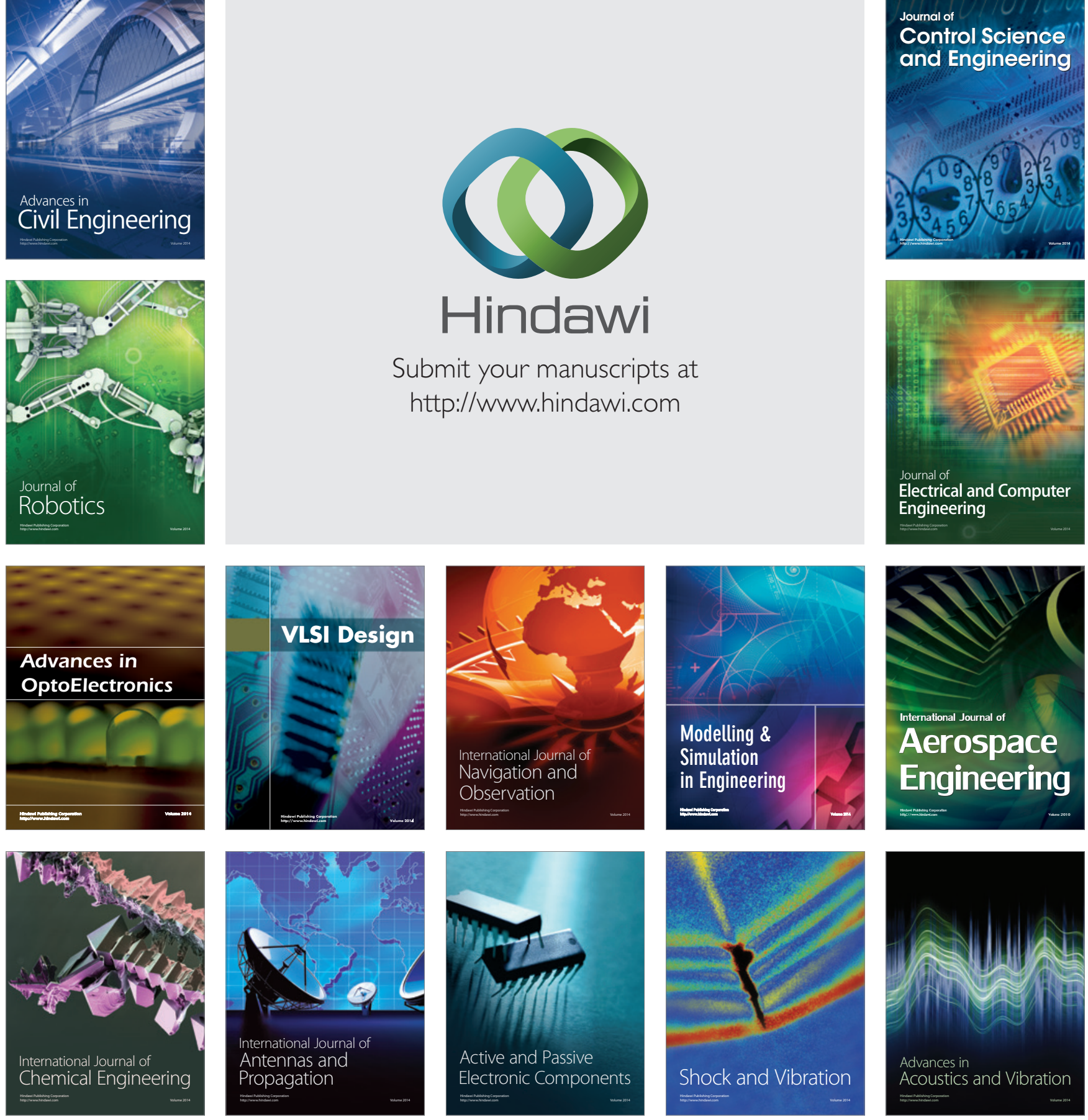